\title{
RELIABILITY ANALYSIS OF CFT COLUMN BY SIMULATION
}

\section{APPROCH}

\author{
Chethan Kumar. $\mathbf{S}^{1}$, Khalid Nayaz Khan ${ }^{2}$, N.S.Kumar ${ }^{3}$ \\ ${ }^{I}$ Final Year Student, Department of Civil Engineering, Ghousia College of Engineering, Karnataka, India \\ ${ }^{2}$ Associate Professor, Department of Civil Engineering, Ghousia College of Engineering, Karnataka, India \\ ${ }^{3}$ Professor and Director (R\&D-Civil Engineering.), Department of Civil Engineering, Ghousia College of \\ Engineering, Karnataka, India
}

\begin{abstract}
To gain clear knowledge about the effect of random properties of CFT, reliability analysis of CFT is carried out in the present study. The parametric influences on the CFT should be studied.In most of the previous research, CFT are restricted to a deterministic approach. For reliability analysis, a performance function is used to define. The capacity of the structure was obtained by Experimental results conducted. Resistance function for the structure formulated based on the provisions of Euro code on composite structures. Then the reliability of CFT by using Monte Carlo simulation is carried out. Form the research, it is concluded that fluctuations of such parameters has significant influences on structural reliability of the CFT.
\end{abstract}

Keywords: CFT, Monte Carlo Simulation, Reliability.

\section{INTRODUCTION}

In all the developed countries the composite constructions is popular and in the recent years the composite construction gaining its importance in India. The level of handling in low-rise residential and commercial constructions is increasing since few decades, owing to the merits such as highest strength-to-weight ratio thereby reducing transportation and handling costs. The composite constructions offer high flexibility in design leading to wider range of applications.

The idea of filling concrete into the hallow steel tubes gives several structural advantages such as enhancement in strength due to confinement of concrete, reduction in dead load, subsequent decrease in material and quick construction compared to usual methods. The strength enhancement is found to be depending on factors such as cross-sectional shape, slenderness ratio, flat width or diameter to thickness ratio or thickness of steel tube, concrete core strength and local buckling behavior of steel tube.

Fred Moses summarizes the steps involved in Reliability analysis as follows:

$>$ Collection and statistical analysis of data on basic variable. Defining the probability distribution of each variable in terms of mean values, standard deviation and type of probability distribution.

$>$ Statistical study of strength (resistance) of members and establishing their statistics.

$>$ Reliability analysis \& determination of reliability index, $\beta$ for the members designed as per the present code for each load combination.
$>$ Determination of the probability of failure through the reliability index.

Numerical procedures viz, the Monte Carlo Simulation, the First Order Reliability Method (FORM) and Second Order Reliability Method (SORM) are used to calculate the probability of failure in Reliability based design optimization .Large numbers of samples were used to derive the probability of failure in crude Monte Carlo method or importance sampling method.

Probability of failure is calculated by using some numerical methods such as FORM, SORM, Monte Carlo simulation \& etc. in direct Monte Carlo simulation or importance sampling method probability of failure is obtained by using the several large number of test data . In FORM, SORM or AMVM, a additional procedure is used locate the most probable point of failure.

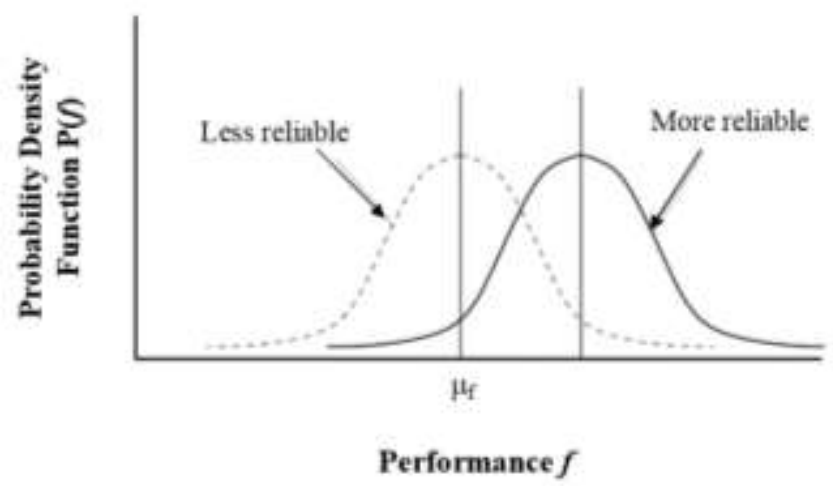

Fig:1 RBDO design strategy 


\section{EXPERIMENTAL RESULTS}

Table: 1 Result

\begin{tabular}{|c|c|c|c|c|c|c|}
\hline $\begin{array}{l}\text { Exp } \\
\text { no }\end{array}$ & $\begin{array}{l}\mathrm{D} \\
\mathrm{mm}\end{array}$ & $\begin{array}{l}\mathrm{T} \\
\mathrm{mm}\end{array}$ & $\begin{array}{l}\mathrm{L} \\
\mathrm{mm}\end{array}$ & $\mathrm{f}_{\mathrm{ck}}$ & $\mathrm{f}$ & $\mathrm{P}_{\mathrm{u}}$ \\
\hline 1 & 33.7 & 2.6 & 300 & 23.93 & 310 & 123 \\
\hline 2 & 33.7 & 2.6 & 300 & 28.06 & 310 & 138 \\
\hline 3 & 33.7 & 2.6 & 300 & 29.01 & 310 & 135 \\
\hline 4 & 33.7 & 3.2 & 300 & 23.93 & 310 & 145 \\
\hline 5 & 33.7 & 3.2 & 300 & 28.06 & 310 & 147 \\
\hline 6 & 33.7 & 3.2 & 300 & 29.01 & 310 & 143 \\
\hline 7 & 33.7 & 4 & 300 & 23.93 & 310 & 172 \\
\hline 8 & 33.7 & 4 & 300 & 28.06 & 310 & 178 \\
\hline 9 & 33.7 & 4 & 300 & 29.01 & 310 & 175 \\
\hline 10 & 42.4 & 2.6 & 300 & 23.93 & 310 & 165 \\
\hline 11 & 42.4 & 2.6 & 300 & 28.06 & 310 & 175 \\
\hline 12 & 42.4 & 2.6 & 300 & 29.01 & 310 & 169 \\
\hline 13 & 42.4 & 3.2 & 300 & 23.93 & 310 & 182 \\
\hline 14 & 42.4 & 3.2 & 300 & 28.06 & 310 & 190 \\
\hline 15 & 42.4 & 3.2 & 300 & 29.01 & 310 & 185 \\
\hline 16 & 42.4 & 4 & 300 & 23.93 & 310 & 210 \\
\hline 17 & 42.4 & 4 & 300 & 28.06 & 310 & 221 \\
\hline 18 & 42.4 & 4 & 300 & 29.01 & 310 & 216 \\
\hline 19 & 48.3 & 2.6 & 300 & 23.93 & 310 & 190 \\
\hline 20 & 48.3 & 2.6 & 300 & 28.06 & 310 & 198 \\
\hline 21 & 48.3 & 2.6 & 300 & 29.01 & 310 & 191 \\
\hline 22 & 48.3 & 3.2 & 300 & 23.93 & 310 & 198 \\
\hline 23 & 48.3 & 3.2 & 300 & 28.06 & 310 & 203 \\
\hline 24 & 48.3 & 3.2 & 300 & 29.01 & 310 & 200 \\
\hline 25 & 48.3 & 4 & 300 & 23.93 & 310 & 223 \\
\hline 26 & 48.3 & 4 & 300 & 28.06 & 310 & 230 \\
\hline 27 & 48.3 & 4 & 300 & 29.01 & 310 & 228 \\
\hline
\end{tabular}

\section{SIMULATION-BASED}

RELIABILITY METHODS

A variety of approximation schemes are employed to compute these probabilities, including sampling techniques based on the Monte Carlo Simulation (MCS) procedure. The Accuracy of MCS estimations increases with increased sampling size, but setting low failure probability levels Pf and dealing with costly constraint functions makes this impractical. Many sampling techniques have been proposed to maintain the advantage of MCS with smaller samples

\section{RELIABILITY ANALYSIS}

According to Eurocode -4

Limit state function is

$$
\mathrm{G}\left(\theta_{\mathrm{E}} \times \mathrm{E}, \theta_{\mathrm{R}} \times \mathrm{R}\right)=\theta_{\mathrm{R}} \times \mathrm{R}-\theta_{\mathrm{E}} \times \mathrm{E}
$$

Where

E- Random variables for action effects.

$\mathrm{R}$ - Random variables for resistance of structural member.

Design buckling resistance of composite column

$\mathrm{R}_{\mathrm{d}}=\mathrm{k}\left\{\left(\mathrm{A}_{\mathrm{s}} \mathrm{F}_{\mathrm{y}} / \gamma_{\mathrm{m}}\right)+\left(0.875 \mathrm{~A}_{\mathrm{c}} \mathrm{F}_{\mathrm{ck}} / \gamma_{\mathrm{c}}\right)\right\}$

$\mathrm{A}_{\mathrm{s}}$ - Area of steel tube,

$\mathrm{A}_{\mathrm{c}}$ - area of concrete,

$\mathrm{F}_{\mathrm{y}}$ - yield strength of steel,

$\mathrm{F}_{\mathrm{ck}}$ - compressive strength of concrete,

$\gamma_{\mathrm{m}} \& \gamma_{\mathrm{c}}-$ partial safety factor,

Table: 2 Statistical parameters of random variables

\begin{tabular}{|c|c|c|c|c|}
\hline $\begin{array}{l}\text { Catego } \\
\text { ry of } \\
\text { Variabl } \\
\text { es }\end{array}$ & Variables & $\begin{array}{l}\text { Distributio } \\
\mathrm{n}\end{array}$ & $\begin{array}{l}\text { Mean } \\
\text { Value } \mu_{\mathrm{x}}\end{array}$ & $\begin{array}{l}\text { Standar } \\
\mathrm{d} \\
\text { Deviati } \\
\text { on } \Sigma_{\mathrm{x}}\end{array}$ \\
\hline \multirow[t]{5}{*}{$\begin{array}{l}\text { Model } \\
\text { Uncert } \\
\text { ainty }\end{array}$} & $\begin{array}{l}\text { Action } \\
\text { Effect } \\
\text { Factor }\left(\Theta_{\mathrm{e}}\right)\end{array}$ & Normal & 1 & 0.10 \\
\hline & $\begin{array}{l}\text { C/S Area } \\
\text { (A) }\end{array}$ & Normal & $\mu_{\mathrm{a}}$ & $0.02 \mu_{\mathrm{a}}$ \\
\hline & $\begin{array}{l}\text { Yield } \\
\text { Strength } \\
\left(\mathrm{F}_{\mathrm{y}}\right)\end{array}$ & $\begin{array}{l}\text { Log- } \\
\text { Normal }\end{array}$ & $\mathrm{F}_{\mathrm{y}}+2 \Sigma_{\mathrm{x}}$ & 30 \\
\hline & $\begin{array}{l}\text { Compressi } \\
\text { ve Strength } \\
\left(\mathrm{F}_{\mathrm{ck}}\right)\end{array}$ & $\begin{array}{l}\text { Log- } \\
\text { Normal }\end{array}$ & $\mathrm{F}_{\mathrm{ck}}+2 \Sigma_{\mathrm{x}}$ & 5 \\
\hline & $\begin{array}{l}\text { Resistance } \\
\text { Factor For } \\
\text { CFT }\left(\Theta_{r}\right)\end{array}$ & Normal & 1.10 & $0.14 \mu_{\mathrm{q}}$ \\
\hline Actions & $\begin{array}{l}\text { Permanent } \\
\text { (G) }\end{array}$ & Normal & $\mathrm{G}_{\mathrm{K}}$ & $0.1 \mu_{\mathrm{g}}$ \\
\hline
\end{tabular}

\subsection{Sample Study}

The reliability of resistance of a CFT column with $\mathrm{D} \times \mathrm{t}=33.7 \mathrm{~mm} \times 2.6 \mathrm{~mm} \&$ length $300 \mathrm{~mm}$ is investigated. The following material properties are employed in the deterministic analysis: compressive strength of unconfined concrete $23.93 \mathrm{~N} / \mathrm{mm}^{2}$, yield strength of steel $310 \mathrm{~N} / \mathrm{mm}^{2}$, and elastic modulus of steel $2000 \mathrm{~s}$ E. GPa.

By solving the above by Monte Carlo simulation method in COMREL 9 the obtained reliability index is $\beta$ is -1.579 . The corresponding probability of failure is obtained by using $\mathrm{Z}$ table. $P_{\mathrm{f}}=0.05821$, therefore the reliability of the CFT column subjected to axial load is $\mathrm{R}=1-\mathrm{P}_{\mathrm{f}}$, i.e. $\mathrm{R}=94.179 \%$. 
Table: 3 Reliability Analysis

\begin{tabular}{|c|c|c|c|c|c|}
\hline Exp no & $\mathrm{P}_{\mathrm{u}}$ & MCS $\boldsymbol{\beta}$ & $P_{f}$ & $\mathrm{R}$ & $\mathrm{R}$ in $\%$ \\
\hline 1 & 123 & -1.579 & 0.0582 & 0.9418 & 94.179 \\
\hline 2 & 138 & -2.125 & 0.017 & 0.983 & 98.3 \\
\hline 3 & 135 & -1.988 & 0.0239 & 0.9762 & 97.615 \\
\hline 4 & 145 & -1.617 & 0.0537 & 0.9463 & 94.63 \\
\hline 5 & 147 & -1.63 & 0.0516 & 0.9485 & 94.845 \\
\hline 6 & 143 & -1.459 & 0.0722 & 0.9279 & 92.785 \\
\hline 7 & 172 & -1.628 & 0.0516 & 0.9485 & 94.845 \\
\hline 8 & 178 & -1.77 & 0.0384 & 0.9616 & 96.164 \\
\hline 9 & 175 & -1.666 & 0.0485 & 0.9515 & 95.154 \\
\hline 10 & 165 & -1.61 & 0.0537 & 0.9463 & 94.63 \\
\hline 11 & 175 & -1.832 & 0.0336 & 0.9664 & 96.638 \\
\hline 12 & 169 & -1.61 & 0.0505 & 0.9495 & 94.95 \\
\hline 13 & 182 & -1.21 & 0.1131 & 0.8869 & 88.686 \\
\hline 14 & 190 & -1.451 & 0.0735 & 0.9265 & 92.647 \\
\hline 15 & 185 & -1.276 & 0.102 & 0.898 & 89.796 \\
\hline 16 & 210 & -1.134 & 0.1292 & 0.8708 & 87.076 \\
\hline 17 & 221 & -1.371 & 0.8534 & 0.1466 & 14.66 \\
\hline 18 & 216 & -1.215 & 0.1131 & 0.8869 & 88.686 \\
\hline 19 & 190 & -1.476 & 0.7078 & 0.2922 & 29.22 \\
\hline 20 & 198 & -1.578 & 0.0582 & 0.9418 & 94.179 \\
\hline 21 & 191 & -1.01 & 0.1563 & 0.8438 & 84.375 \\
\hline 22 & 198 & -0.815 & 0.209 & 0.791 & 79.103 \\
\hline 23 & 203 & -0.857 & 0.1977 & 0.8023 & 80.234 \\
\hline 24 & 200 & -0.737 & 0.2327 & 0.7673 & 76.73 \\
\hline 25 & 223 & -0.521 & 0.2451 & 0.7549 & 75.49 \\
\hline 26 & 230 & -0.622 & 0.2676 & 0.7324 & 73.237 \\
\hline 27 & 228 & -0.549 & 0.2912 & 0.7088 & 70.884 \\
\hline
\end{tabular}

\section{RESULTS}

For the analysis of results obtained Taguchi's L9 combination considered. Results for the constant length various diameters, thickness, grade of concrete are considered as per Taguchi level-3 design (Table-4) with 3factors.

Table: 4 Taguchi's L 9 Orthogonal Array

\begin{tabular}{|l|l|l|l|}
\hline Sl No & Dia & Thickness & $\begin{array}{l}\text { Grade of } \\
\text { Concrete }\end{array}$ \\
\hline 1 & 1 & 1 & 1 \\
\hline 2 & 1 & 2 & 2 \\
\hline 3 & 1 & 3 & 3 \\
\hline 4 & 2 & 1 & 2 \\
\hline 5 & 2 & 2 & 3 \\
\hline 6 & 2 & 3 & 1 \\
\hline 7 & 3 & 1 & 3 \\
\hline 8 & 3 & 2 & 1 \\
\hline 9 & 3 & 3 & 2 \\
\hline
\end{tabular}

\begin{tabular}{|l|l|l|l|l|l|}
\hline $\begin{array}{l}\text { Exp } \\
\text { no }\end{array}$ & $\mathrm{P}_{\mathrm{u}}$ & $\begin{array}{l}\mathrm{MCS} \\
\boldsymbol{\beta}\end{array}$ & $\mathrm{P}_{\mathrm{f}}$ & $\mathrm{R}$ & $\mathrm{R}$ in \% \\
\hline 1 & 123 & -1.579 & 0.05821 & 0.94179 & 94.179 \\
\hline 5 & 147 & -1.63 & 0.05155 & 0.94845 & 94.845 \\
\hline 9 & 175 & -1.666 & 0.04846 & 0.95154 & 95.154 \\
\hline 11 & 175 & -1.832 & 0.03362 & 0.96638 & 96.638 \\
\hline 15 & 185 & -1.276 & 0.10204 & 0.89796 & 89.796 \\
\hline 16 & 210 & -1.134 & 0.12924 & 0.87076 & 87.076 \\
\hline 21 & 191 & -1.01 & 0.15625 & 0.84375 & 84.375 \\
\hline 22 & 198 & -0.815 & 0.20897 & 0.79103 & 79.103 \\
\hline 26 & 230 & -0.622 & 0.26763 & 0.73237 & 73.237 \\
\hline
\end{tabular}

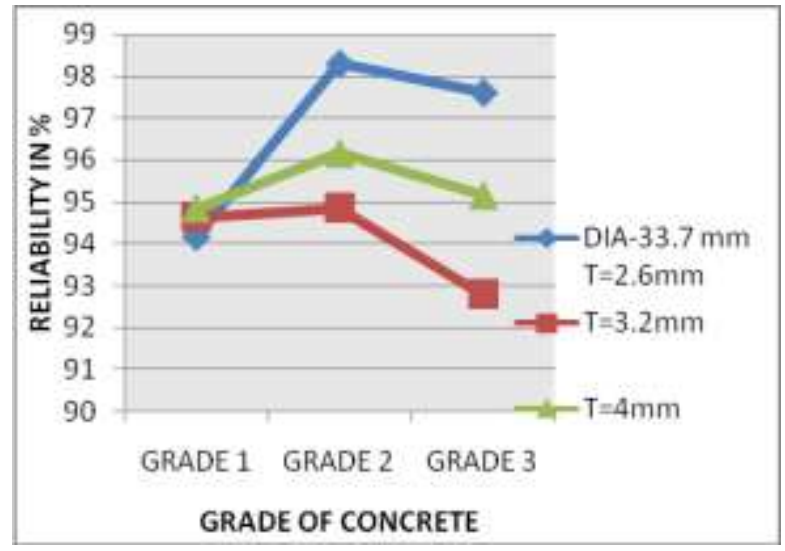

Fig: 2 Grade of Concrete V/S Reliability

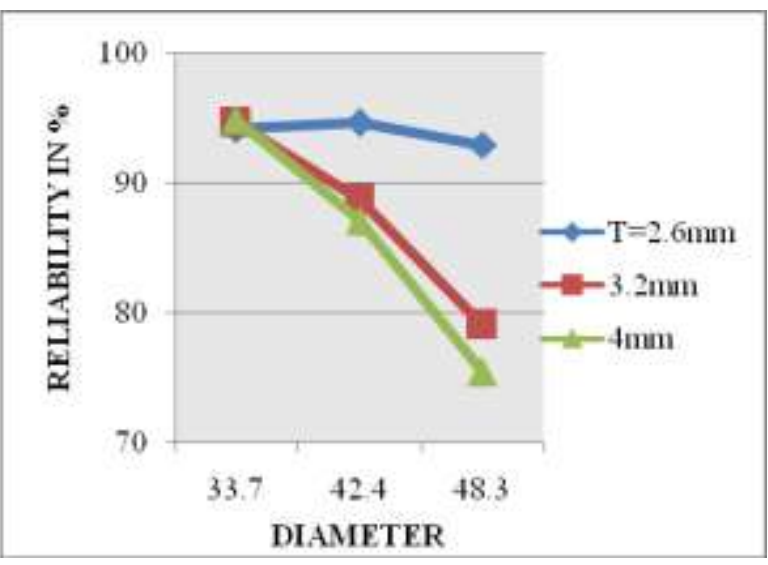

Fig: 3 Diameter V/S Reliability

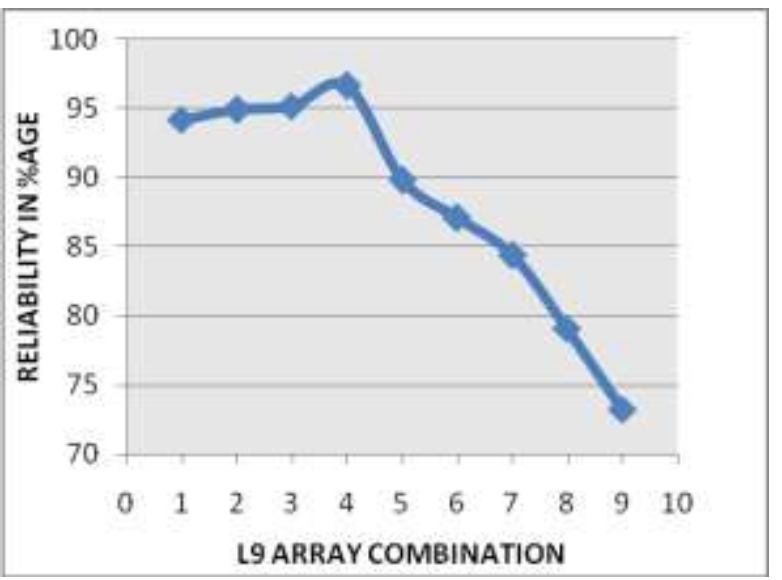

Fig: 4 Taguchi's Array Combination V/S Reliability 


\section{CONCLUSION}

$>$ As the Grade of concrete is increased, the safety of the designed element also increased.This was due to the fact that the load carrying capacity of the designed section is a function of concrete strength.(Fig 2)

$>$ Generally, as diameter of the section increases, the safety of the designed section decreases. (Fig 3)

$>$ It can be observed from the table-4 L9 array, that the maximum reliability obtained is $96.638 \%$ for $\mathrm{D}_{2}, \mathrm{~T}_{1}$, and $\mathrm{G}_{2}$ combination for a constant length.

$>$ From fig 4 it can be conclude that the optimum combination obtained was $\mathrm{D}_{2}, \mathrm{~T}_{1}$, and $\mathrm{G}_{2}$.

\section{REFERENCES}

[1] Anukal C. and Mahadevan S. (2005), 'First-Order Approximation Methods in Reliability-Based Design Optimization' Journal of Mechanical Design, Vol. 127, pp. 851-857.

[2] Fred Moses, (1997) 'Problems and prospects of reliability-based optimization', Journal of Engineering Structures, Vol. 19 No. 4 pp. 293-301.

[3] Ranganathan. R. (1990), "Reliability Analysis and Design of Structures" Tata McGraw Hill.

[4] Ditlevsen, O.; Madsen, H. (1996): Structural Reliability Methods, John Wiley \& Sons.

[5] Eurocode 4, EN 1994-1-1: 2004. Design of composite steel and concrete structures-Part11:General rules and rules for buildings. CEN: Brussels.

[6] JCSS (Joint Committee on Structural Safety). 2000. Probabilistic Model Code: Part 1 - Basis of design. 12th draft.

[7] RCP - Reliability Consulting Programs STRUREL, A Structural Reliability Analysis Program System, COMREL \& SYSREL Users Manual RCP Consult, München 1995

[8] Melchers, R.E. 1999. Structural reliability analysis and prediction, Chichester, JohnWiley.

[9] Calibration of Reliability Elements for a Column, Milan Holicky, Jana Markova JCSS Workshop on Reliability Based Code Calibration.

[10] Reliability analysis of a reinforced concrete column designed according to the Eurocodes.Holický, Milan / Vrouwenvelder, Ton,IABSE reports = Rapports AIPC = IVBH Bericht pp251-264.

[11] Kavya.M.S, Dr. N.S. Kumar \& Dr. Mohamed Ilyas Anjum "Behavior Of Composite Circular Steel Columns In filled With Fiber Reinforced Concrete Subjected To Monotonic Loading” M.Tech dissertation 2012

[12] Ramesh Muthu.D\& Dr.N.S.Kumar (2013) "Experimental Investigation on Concrete Filled Stainless Steel Tubular (Cfsst) Short Columns under Monotonic Loading". M.Tech dissertation 2012

[13] AV. Raviprakash , A. Adhithya Plato Sidharth, B. Prabu ‘N. Alagumurthi "Structural Reliability of Thin Plates with Random Geometrical Imperfections Subjected to Uniform Axial Compression" Jordan Journal of Mechanical and Industrial Engineering pp 270-278
[14] AntanasKudzys, RomualdasKliukas "Reliability Index Design In Reinforced Concrete Structures Of Annular Cross Sections" 